Ann. Zootech., I977, 26 (4), 62I-625.

\title{
Note
}

\section{Influences sur la puberté de la truie de sa période de naissance et de sa durée d'allaitement}

\author{
Sylvaine CARREZ, F. TREIL, P.-H. DUÉE et A. AUMAITRE \\ Station de Recherches sur l'Elevage des Porcs \\ Centre national de Recherches zootechniques, I.N.R.A., \\ $7835^{\circ}$ Jouy en Josas (France)
}

\begin{abstract}
Résumé
Une étude statistique a été entreprise sur 362 porcelets femclles qui diffèrent par l'âge au sevrage (Io, 2x, 35 jours) et par la période de naissance. La réduction de la durée d’allaitement ne modifie pas l'apparition du premier oestrus. Il existe une relation linéaire entre le poids à deux mois d'une part, la vitesse de croissance après deux mois d'autre part, et l'âge à la puberté (respectivement, $r=-0,13 ; \gamma=-0,47)$. Par ailleurs, les animaux qui naissent entre mai et octobre ont une puberté plus précoce ( 243 jours) que ceux nés durant l'autre période de l'année ( 253 jours). Cet effet n'est cependant pas expliqué par le poids à deux mois ou la vitesse de croissance prépubertaire.
\end{abstract}

\section{Introduction}

La productivité de la truie est conditionnée en partie par l'âge auquel apparaît la fonction de reproduction. Or, l'apparition de la puberté fait l'objet d'estimations généralement très variables; de nombreux facteurs (génotype, nutrition ou milieu) peuvent expliquer cette hétérogénéité de l'âge au premier oestrus (RERAT et DUÉE, I976), mais leurs effets sont souvent contradictoires.

Dans le domaine nutritionnel, on a cependant remarqué que certains cas de carence globale ou spécifique, pendant la période de croissance, retardaient la puberté (ÉTIENNE et DUÉE, I 973; CunNingham et al., I974); on peut alors se demander si la vitesse de croissance, modifiée dans le cas présent par le niveau des apports nutritionnels, ne peut être un des facteurs affectant l'apparition de la maturité sexuelle. 
Dans cette étude, il sera envisagé l'effet des conditions post-natales (période de naissance; durée d'allaitement) sur l'apparition de la puberté, compte tenu des performances de croissance des animaux.

\section{Matériel et méthodes}

Une étude statistique a été entreprise sur 362 porcelets femelles de race Large White qui diffèrent par l'âge au sevrage (Io, 2 I ou 35 jours) ainsi que par le mois de naissance, réparti uniformément sur l'année. Les conditions de logement et d'alimentation sont identiques pour tous les animaux après leur sevrage et la détection du premier oestrus est effectuée à partir de $70 \mathrm{~kg}$ de poids vif par présentation quotidienne au verrat.

Les effets de la durée d'allaitement et de la période de naissance, après regroupement des naissances en deux périodes de six mois, "froide " (de novembre à avril) et "chaude " (de mai à octobre), sur l'apparition de la puberté ont été étudiés en comparant les valeurs moyennes par analyse de variance. L'étude des fréquences de distribution de l'âge et du poids à la puberté a été effectuée par le test 2 î (ARBONNIER, I 966). Enfin, on a procédé au calcul des corrélations linéaires entre le poids à âges fixes (naissance, 2I, 35 ou 60 jours) ou la vitesse de croissance de deux mois à la puberté et 1'âge au premier oestrus.

\section{Résultats}

\section{I. - Infuence de la durée d'allaitement sur l'âge et le poids à la puberté}

L'étude porte sur un effectif de 334 cochettes contemporaines, réparties inégalement suivant 3 durées d'allaitement : ro jours ( 57 animaux); 2 I jours ( 79 animaux) 35 jours (Ig8 animaux). Les valeurs moyennes de l'âge et du poids à la puberté, comparées par analyse de variance ne font ressortir aucune différence significative entre les trois durées d'allaitement. On peut toutefois remarquer l'âge élevé des animaux à la maturité sexuelle. L'analyse de la distribution des fréquences (fig. I) souligne une tendance, pour les femelles sevrées à ro jours, à atteindre la puberté plus tardivement : la fréquence maximale se situe entre 260 et 280 jours contre 240 et 260 jours pour une durée d'allaitement plus longue ( 2 I ou 35 jours). Le poids moyen à la puberté est alors plus faible $(95$ à $105 \mathrm{~kg})$ que celui des animaux sevrés à 2 I ou 35 jours (IO5 à II $5 \mathrm{~kg}$ ). Or, on peut noter que les porcelets sevrés très précocément (Io jours) accusent un retard de croissance immédiat, partiellement compensé à deux mois d'âge, qui toutefois persiste encore à la puberté et pourrait expliquer un âge légèrement plus élevé au premier oestrus.

\section{2. - Relations entre le poids à âges fixes ou la vitesse de croissance prépubertaire et l'âge à la puberté}

Dans chaque classe d'âge au sevrage, il n'a pas été établi de corrélation linéaire significative entre le poids à la naissance des animaux et leur âge à la puberté. Par contre, de telles liaisons existent, dans la classe correspondant au sevrage 
le plus précoce, entre le poids à $2 \mathbf{I}$ ou 35 jours et l'âge au premier oestrus (à $2 \mathbf{I}$ jours : $r=-0,30$; à 35 jours : $r=-0,28 \mathrm{P}<0,05)$.

Ceci soulignerait un effet précoce des conditions nutritionnelles sur 1'âge à la puberté, qui est mis en évidence lors d'une durée d'allaitement réduite. Quoi qu'il en soit, pour l'ensemble des animaux (362), il existe une corrélation linéaire,

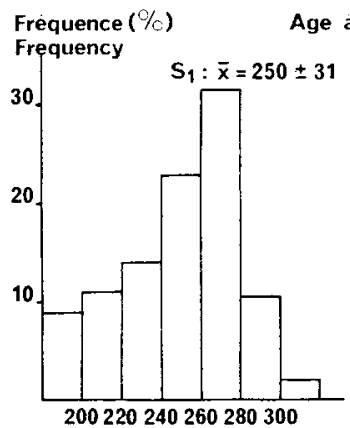

Age a la puberte (jours)

Age at puberty (days)
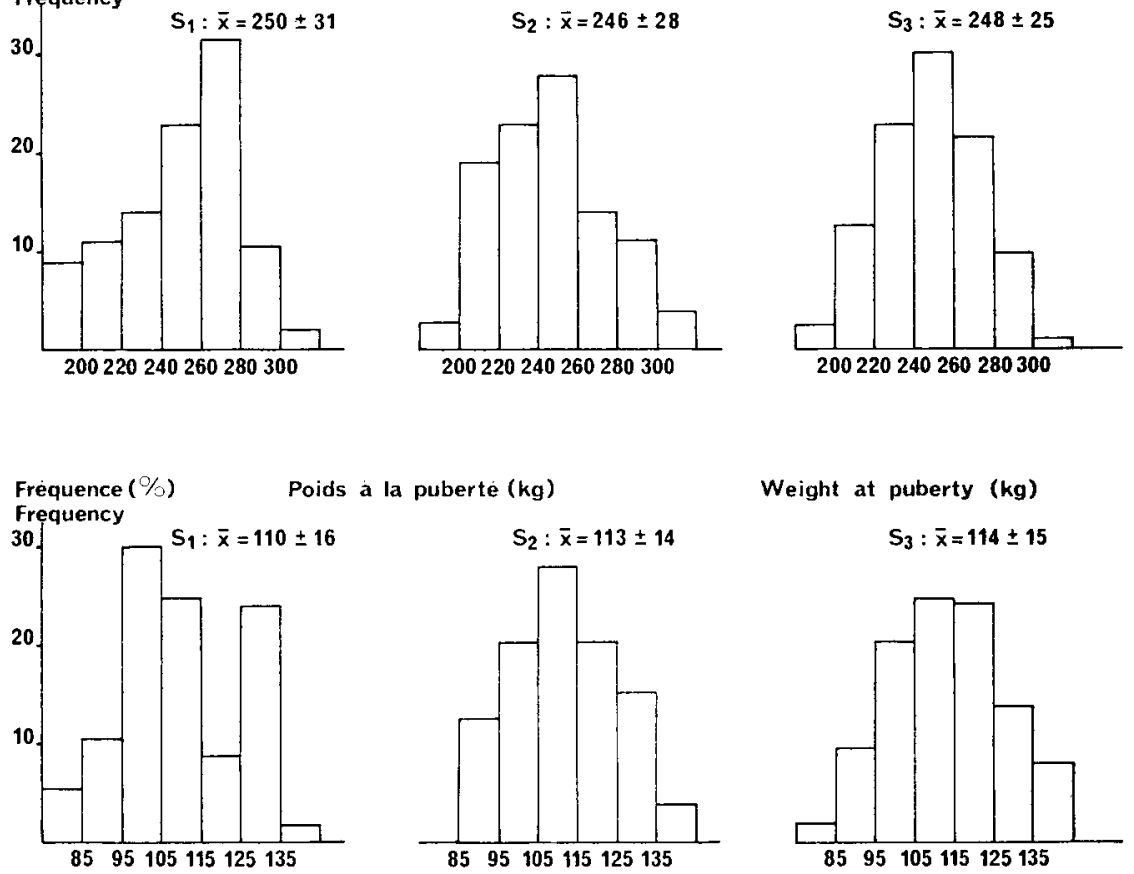

FIG. I. - Distribution des fréquences de l'âge et du poids à la puberté selon l'âge au sevrage Distribution of age and weight frequencies at puberty according to age at weaning.

$\mathrm{S}_{1}$ : Sevrage à ro jours - Weaning on day $\mathrm{Io}$.

$\mathrm{S}_{2}$ : Sevrage à $2 \mathrm{I}$ jours - Weaning on day $2 x$.

$\mathrm{S}_{3}$ : Sevrage à 35 jours - Weaning on day 35 .

faible il est vrai, entre le poids à deux mois et l'âge à la puberté $(r=-0, I 3$; $\mathrm{P}<0, \mathrm{OI})$.

Par ailleurs, la vitesse de croissance des animaux entre deux mois et l'âge au premier ostrus détecté ( $x$, en grammes par jour), est reliée linéairement à 1'âge à la puberté ( $y$, en jours).

$$
y=-0, \mathrm{I} 8 x+340,35 ; \quad r=-0,47(\mathrm{P}<0, \text { oor })
$$

Ainsi, plus la vitesse de croissance est élevée, plus l'animal est jeune à la puberté. L'intensité de croissance prépubertaire semble donc un facteur non négligeable dans le déclenchement de la maturité sexuelle. 


\section{3. - Influence de la période de naissance sur l'âge et le poids à la puberté}

Les données consignées dans le tableau I indiquent que, dans le cas d'une naissance en période chaude (de mai à octobre), la maturité sexuelle est plus précoce, d'environ ro jours. Pour ce qui concerne l'effet de la saison de naissance sur la puberté, les résultats bibliographiques sont contradictoires (RERAT et DUÉE, r976). Il est cependant établi que l'intensité d'éclairement peut modifier l'appa-

\section{TABLEAU I}

Effet de la période de naissance sur la puberté

Effect of birth period on puberty

\begin{tabular}{|c|c|c|c|c|}
\hline $\begin{array}{c}\text { Période } \\
\text { Period }\end{array}$ & $\begin{array}{l}\text { Effectif } \\
\text { Number }\end{array}$ & $\begin{array}{l}\text { Age à la puberté } \\
\text { (jours) } \\
\text { Age at puberty } \\
\text { (days) }\end{array}$ & $\begin{array}{l}\text { Poids à la puberté } \\
\text { (kg) } \\
\text { Weight at puberty } \\
(k g)\end{array}$ & $\begin{array}{c}\text { Poids à a mois } \\
(\mathrm{kg}) \\
\text { Weight at } 2 \text { months } \\
(\mathrm{kg})\end{array}$ \\
\hline $\begin{array}{l}\text { Froide - Cold . . } \\
\quad \text { (nov.-avr.) } \\
\begin{array}{l}\text { Chaude - Warm . . } \\
\text { (mai-oct.) }\end{array}\end{array}$ & I 67 & $\begin{array}{c}253, \mathrm{I} \pm 2, \mathrm{O}(\mathrm{I}) \\
(* *) \\
242,6 \pm \mathrm{I}, 9\end{array}$ & $\begin{array}{c}\text { I I } 6,0 \pm 1,2 \\
(* *) \\
\text { I I I,5 } \pm \text { I , } 0\end{array}$ & I $8,0 \pm 0,2$ \\
\hline
\end{tabular}

(r) Moyenne \pm écart-type de la moyenne. Mean value \pm mean standard deviation.

(**) : $(\mathrm{P}<\mathrm{o}, \mathrm{or})$.

rition de la puberté (HACkER et al., I974; I976) et principalement pendant le jeune âge (MarTinat et al., I970).

On ne peut toutefois expliquer l'écart de dix jours au premier oestrus entre les femelles des deux périodes de naissance, ni par une différence dans le poids des animaux à deux mois, ni par une différence d'intensité de croissance entre deux mois et la puberté, qui est comparable dans les deux groupes d'animaux (5Io à 5 I5 g/jour).

\section{Conclusion}

Cette étude montre que la réduction de la durée d'allaitement (de 35 à ro jours) n'est pas préjudiciable à l'apparition de la maturité sexuelle. Par ailleurs, la vitesse de croissance de deux mois à la puberté est liée négativement avec l'âge au premier oestrus, confirmant par là même l'importance des conđitions nutritionnelles durant cette période.

De plus, les conditions d'élevage pourraient intervenir précocément (avant 2 mois d'âge), si l'on considère les corrélations établies dans le groupe d'animaux 
sevrés à Io jours. Toutefois, ces paramètres liés à la vitesse de développement des jeunes femelles sont insuffisants pour expliquer l'effet de la période de naissance constaté dans cette étude, ce qui souligne la complexité des phénomènes induisant la maturité sexuelle.

Accepté pour publication en septembre 1977 .

\section{Summary}

Effects of birth period and suckling length on the onset of puberty in gilts

A statistical study was made with 362 female piglets differing by the age at weaning (Io, 2I, 35 days) and the birth period. Redurtion of the suckling length did not modify the onset of Ist oestrus. Weight at two months, on the one hand and growth rate between the age of two months and puberty, on the other, were negatively correlated with the age at puberty $(r=-0,13$; $r=-0,47$, respectively). Furthermore, animals, which were born between May and October exhibited earlier puberty (243 days) than those born during the other months of the year (253 days). However, this effect is not explained either by the weight at two months or by the prepuberal growth rate.

\section{Références bibliographiques}

Arbonnier P., ig66. L'analyse de 1'information : Aperçu théorique et application à la loi multinomiale. Ann. Sci. Forest., 23, 951-Ior7.

Cunningham P. J., Naber C. H., Zimmerman D. R., Peo E. R., Jr., I974. Influence of nutritional regime on age at puberty in gilts. J. anim. Sci. 39,63-67.

FiTIENNE M., DUÉE P. H., r973. Effets respectifs des niveaux alimentaires pendant la croissance et le premier mois de gestation sur les performances de reproduction chez la truie nullipare : résultats préliminaires. Ann. Zootech., 22, 453-462.

HACKER R, R., KING G. J., BEARSS W. H., I974. Effects of complete darkness on growth and reproduction in gilts. $J$. anim. Sci., 39, I55 (abstr.).

HACKER R. R., King G. J., Smith V. G., 1976. Effects of 6 and 58 hr light on reproduction in gilts. J. anim. Sci., 43, 228 (Abstr.).

Martinat F., Legault C., Du Mesnil du Buisson F., Olifivier L., Signoret J. P., I97o. Itude des retards de puberté chez la truie. Journées Rech. Porcine en France, I.N.R.A. - I.T.P. éd., Paris, 47-54.

RERAT A., DUIEE P. H., I976. Conditions d'élevage et alimentation chez la jeune truie : influence sur la carrière ultérieure de l'animal. in La conduite du troupeau de reproducteurs porcins., édité par Études et Formation Vétérinaires, I5-24. 